\title{
Comparative Evaluation of the Antioxidant Effects of Oregano on Bleached Enamel Using Energy-dispersive X-ray Spectroscopy Analysis: An In Vitro Study
}

\author{
Bharath Naga Reddy ${ }^{1}$, Sabari Murugesan ${ }^{2}$, Shanmugavadivel Gandhi ${ }^{3}$, Sujith Rajamohan ${ }^{4}$, Buggaveeti Pradeep Kumar ${ }^{5}$, \\ Purushotham Mohankumar ${ }^{6}$
}

\begin{abstract}
Aim and objective: The aim of this study was to evaluate the efficacy of novel antioxidant oregano against conventional antioxidants sodium ascorbate and green tea through comparison of the baseline weight percentage (wt\%) of minerals prior to bleaching with values after bleaching protocols and antioxidant application by employing energy-dispersive X-ray spectroscopy analysis (EDAX).

Materials and methods: Thirty noncarious, freshly extracted human permanent maxillary incisors without any visible defects were selected as samples. They were divided into three groups $(n=10)$ based on the antioxidant treatment received as follows: group I-10\% sodium ascorbate solution, group II-10\% green tea solution, and group III-5\% oregano solution. $35 \%$ hydrogen peroxide was employed for bleaching. The wt $\%$ of teeth was calculated at three time intervals. The initial wt\% was calculated prior to the bleaching procedure. The second one was calculated after subjecting to bleaching protocols, and final one was calculated after antioxidant treatment using EDAX.

Results: There was a statistically significant decrease in the calcium/phosphorus ratio for the bleached samples in comparison with the sound enamel. There was a comparable and significant increase in the calcium/phosphorus ratio values after application of the antioxidant with insignificant difference among the antioxidants evaluated in the study.

Conclusion:Treatment with antioxidants helped in the reversal of mineral loss, which occurred due to the bleaching procedure while the efficacy of $10 \%$ oregano on reversal of the calcium and phosphorus ratio was found to comparable to that of $10 \%$ sodium ascorbate and green tea.

Clinical significance: Application of antioxidants after bleaching reduced the time delay in performing the adhesive procedures without compromising their clinical efficacy.

Keywords: Antioxidants, Bleaching agents, Green tea, Oregano, Sodium ascorbate.

The Journal of Contemporary Dental Practice (2020): 10.5005/jp-journals-10024-2912
\end{abstract}

\section{INTRODUCTION}

Tooth discoloration can be broadly categorized as extrinsic and intrinsic discoloration. Intrinsic discoloration occurs predominantly during the developmental stages of tooth. It includes amelogenesis imperfecta, dentinogenesis imperfecta, and enamel hypoplasia.

Drug-induced discoloration occurs due to the consumption of drugs during the formative stages of enamel and dentin. The acquired stains occur due to variety of causes, which can be grouped as microbial, dietary, and lifestyle changes. It predominantly affects the enamel and dentin components of hard tissues. A chemical interaction occurs between the acquired enamel pellicle, carbohydrate component of diet, and chromogenic microbes to produce the discoloration. ${ }^{1}$

The selection of bleaching as a treatment option depends upon the severity of discoloration. In mild to moderate cases, it is selected as a primary treatment option while in severe cases its selected as a secondary option to veneers. ${ }^{2}$

In the esthetic era, the bleaching procedure becomes an essential treatment option rather than a sophisticated option. The outcome of bleaching must be free from complication. The success of dental bleaching relies predominantly on the cautious handling of chemicals such hydrogen peroxide and carbamide peroxide. Hydrogen peroxide available in various concentrations exerts its action by two major mechanisms: (a) release of free radicals and (b) redox processes all leading to the disintegration of pigments. ${ }^{3}$

\begin{abstract}
${ }^{1,4}$ Department of Conservative Dentistry and Endodontics, Adhiparasakthi Dental College and Hospital, Melmaruvathur, Tamil Nadu, India

${ }^{2}$ Department of Restorative Dental Sciences, College of Dentistry, Jazan University, Jazan, Kingdom of Saudi Arabia

${ }^{3}$ Department of Pedodontics and Preventive Dentistry, Sri Venkateshwaraa Dental College, Puducherry, India

${ }^{5}$ Department of Conservative Dentistry and Endodontics, Mamata Dental College and Hospital, Khammam, Telangana, India

${ }^{6}$ Department of Conservative Dentistry and Endodontics, Sathyabama Dental College and Hospital, Chennai, Tamil Nadu, India

Corresponding Author: Bharath Naga Reddy, Department of Conservative Dentistry and Endodontics, Adhiparasakthi Dental College and Hospital, Melmaruvathur, Tamil Nadu, India, Phone: +91 9444792260, e-mail: drbharathendo999@gmail.com
\end{abstract}

How to cite this article: Reddy BN, Murugesan S, Gandhi S, et al. Comparative Evaluation of the Antioxidant Effects of Oregano on Bleached Enamel Using Energy-dispersive X-ray Spectroscopy Analysis: An In Vitro Study. J Contemp Dent Pract 2020;21(10):1170-1175.

Source of support: Nil

Conflict of interest: None

Application of bleaching agents not only changes the micromorphology of enamel and dentin but also their mineral

(c) The Author(s). 2020 Open Access This article is distributed under the terms of the Creative Commons Attribution 4.0 International License (https://creativecommons. org/licenses/by-nc/4.0/), which permits unrestricted use, distribution, and non-commercial reproduction in any medium, provided you give appropriate credit to the original author(s) and the source, provide a link to the Creative Commons license, and indicate if changes were made. The Creative Commons Public Domain Dedication waiver (http://creativecommons.org/publicdomain/zero/1.0/) applies to the data made available in this article, unless otherwise stated. 
content. The primary complication related to bleaching leads to dentin hypersensitivity and gingival irritation. ${ }^{4}$

The series of change that occurs in the enamel after application of hydrogen peroxide occurs due to drop in $\mathrm{pH}$ from 5.2 to 4 , which increases the diameter of enamel rods leading to formation of macro pores, ${ }^{5-13}$ loss of minerals, ${ }^{14}$ reduced concentration of protein, ${ }^{15,16}$ disintegration of dentin, ${ }^{17}$ alterations in the calciumphosphorus ratio, ${ }^{18}$ increased depth of enamel grooves, ${ }^{19}$ shallow depressions, ${ }^{20}$ and slight erosion. ${ }^{21}$

The persistent peroxide effect increases the solubility of inorganic ions and alters its original ratio, thereby decreasing the $\mathrm{Ca}-\mathrm{P}$ ratio of enamel. Hence, it is mandatory to prevent the alteration of the $\mathrm{Ca}-\mathrm{P}$ ratio by nullifying the persistent peroxide effect. Previous studies have reported the action of the persistent peroxide effect counteracted with the immediate application of antioxidants. ${ }^{22,23}$

The latency period following the bleaching procedures ranges from 1 to 3 weeks, which is required to minimize the persistent peroxide effect that occurs due to the usage of hydrogen peroxide and henceforth to improve the mechanical properties of composite restorative materials, the role of antioxidants becomes vital.

The synergetic effect of composites and bleaching is the need of the hour for esthetic procedures with implementation of the minimal invasive practice.

The conventional antioxidants used for the above purpose include sodium ascorbate obtained from ascorbic acid, which has been proved to be an efficient antioxidant.

The primary mechanism of action is related to the catalytic action for improving the efficacy of conversion of free radicals to polymers leaving fewer amounts of residual free radicals and restoration of redox potential of the substrate.

In order to minimize the adverse effects related to chemical agents recently natural antioxidants such as oregano and green tea were used for the above purpose. The major advantage of these natural antioxidants was their minimal adverse effect on the tooth substrate without compromising its clinical efficacy.

A variety of methods are available in the literature to assess the molecular constituents of dental hard tissues such as infrared spectroscopy, electronic microprobe, Raman spectroscopy, and energy-dispersive X-ray spectroscopy analysis (EDAX). Each of methods operates by different mechanism having its own merits and demerits. The selection of methods is based on the analysis of above concepts and utilized dubiously.

The purpose of this study was to evaluate the efficacy of novel antioxidant oregano against conventional antioxidants sodium ascorbate and green tea through comparison of the baseline wt $\%$ of minerals prior to bleaching with values after bleaching protocols and antioxidant application by employing EDAX.

\section{Materials and Methods}

The study was performed in the Department of Conservative Dentistry and Endodontics at Adhiparasakthi Dental College and Hospital. The support for conduction has been integrated with MAPIMS and the EDAX-related procedures were carried in IIT campus, Chennai, by the principal investigator.

\section{Preparation of Sodium Ascorbate Solution}

Total $10 \mathrm{~g}$ of sodium ascorbate powder was dissolved in $100 \mathrm{~mL}$ of distilled water to obtain $10 \%$ sodium ascorbate solution.

\section{Preparation of $10 \%$ Green Tea Solution}

Green tea leaves were ground into fine powder and packed in a muslin cloth bag for the process of extraction.

The extraction was done using a Soxhlet apparatus using ethanol as solvent and finally distilled. Total $10 \mathrm{~g}$ of green tea extract was dissolved in $100 \mathrm{~mL}$ of distilled water to obtain $10 \%$ green tea solution.

\section{Preparation of $5 \%$ Oregano Solution}

Ground leaves were extracted in $100 \mathrm{~mL}$ of ethanol at room temperature for 24 hours. Then they were concentrated using a rotary evaporator resulting in $5 \%$ oregano solution. ${ }^{24}$

Source of green tea solution and oregano solution was obtained from IMCOPS, Chennai. The study methodology was approved by institutional ethical committee of APDCH.

\section{Teeth Sample Preparation}

Thirty maxillary incisors were collected from the oral surgery department of APDCH. Debridement was done as per the guidelines given by $A A E$ stored in $0.1 \%$ thymol solution at $4^{\circ} \mathrm{C}$ until utilization for a period not extending 1 week.

All the samples were examined under a stereomicroscope at $20 \times$ magnification to rule out any enamel defects or cracks.

The inclusion criteria for the selection of samples were teeth free from caries, restoration extracted due to periodontal considerations with no pretreatment of any chemical agents, and cracks or fractures.

The samples with stains, morphological or structural defects, discolorations, and any visible defects were excluded. The samples were washed in running water to eliminate the thymol residue and stored in artificial saliva.

The storage in artificial saliva was avoided during bleaching and testing protocols.

A total of 30 tooth samples were segregated into three groups $(n=10)$ based on the antioxidant evaluated.

Group I: Treatment subjected with $10 \%$ sodium ascorbate solution.

Group II: Treatment subjected with 10\% green tea solution.

Group III: Treatment subjected with $10 \%, 5 \%$ oregano solution.

\section{Bleaching Protocols}

Labial surfaces of the samples were divided into gingival, middle, and incisal segments. One segment of the tooth sample was subjected to surface varnish application leaving the two segments unprotected for bleaching procedure and antioxidant application.

The bleaching procedure was done in the unprotected areas employing $35 \%$ hydrogen peroxide (Polo office), followed by application of an external light source to activate the bleaching agent for exertion of maximum bleaching efficacy for 15 minutes' activation period (Whitening accelerator and C-Bright-I at $3,000 \mathrm{~mW} / \mathrm{cm}^{2}$ ).

This procedure was repeated after intermittent rinsing with water to have maximum clinical efficacy. After this procedure, the second portion of the labial enamel surface was coated with acidresistant nail varnish.

The third portion that was left uncovered on the labial enamel surface was subjected to the application of antioxidants for a time period of 10 minutes; finally, all the samples were rubbed with a nail polish remover (acetone) and soaked in $99.9 \%$ ethyl alcohol to dry the samples. 
Table 1: Mean calcium, phosphorus, and Ca:P values of sound, bleached, and antioxidant treated bleached (AOTB) enamel surfaces of all the three antioxidant groups

\begin{tabular}{|c|c|c|c|c|c|c|c|c|c|}
\hline \multirow[b]{2}{*}{ Samples } & \multicolumn{3}{|c|}{ Sodium ascorbate } & \multicolumn{3}{|c|}{ Green tea } & \multicolumn{3}{|c|}{ Oregano } \\
\hline & $\mathrm{Ca} \%$ & $P \%$ & $\mathrm{Ca} / \mathrm{P}$ & $\mathrm{Ca} \%$ & $P \%$ & $\mathrm{Ca} / \mathrm{P}$ & $\mathrm{Ca} \%$ & $P \%$ & $\mathrm{Ca} / \mathrm{P}$ \\
\hline Sound enamel & 37.70 & 17.79 & 2.11 & 38.94 & 17.66 & 2.20 & 36.68 & 17.45 & 2.09 \\
\hline Bleached enamel & 42.98 & 29.08 & 1.47 & 36.50 & 25.45 & 1.42 & 41.07 & 24.56 & 1.70 \\
\hline АОТВ & 38.02 & 17.79 & 2.14 & 38.29 & 17.52 & 2.18 & 37.63 & 17.49 & 2.15 \\
\hline
\end{tabular}

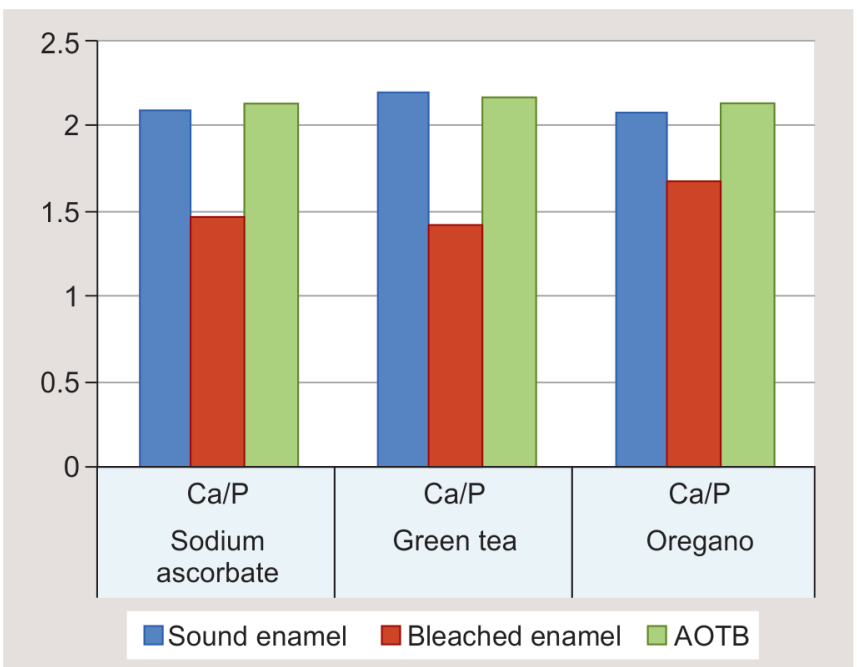

Fig. 1: Antioxidant efficacy. AOTB, antioxidant treated bleached; $\mathrm{Ca}$, calcium; P, phosphorus

\section{Results}

The mineral content of each portion of all the samples were calculated using EDAX. All the samples were placed in an ion sputter to remove the water content from the tooth samples to evaluate the $C a$ and $P$ values.

The baseline values of calcium and phosphorus content were recorded in the sound enamel segment, which was left undisturbed.

The calcium and phosphorus content were recorded in the bleached and antioxidant-treated bleached enamel for all the three antioxidant group. The graphical plots were done for the obtained values using EDAX.

The calcium and phosphorus wt\% were then converted into the $\mathrm{Ca}-\mathrm{P}$ ratio in each portion of three antioxidants and its ratio was calculated from the obtained data.

The data obtained were tabulated and analyzed using SPSS software version 22. The mean calcium/phosphorus ratio data were assessed and found to be normally distributed.

Hence, a parametric test (Tukey "honest significant difference" [HSD] post hoc test) was conducted to assess the statistical significance among sound, bleached, and antioxidant-treated bleached enamel for all the three groups.

In the segment of sound enamel, the $\mathrm{Ca} / \mathrm{P}$ ratio were found to be $2.11,2.20$, and 2.09, respectively for all three antioxidant treated groups as represented in Table 1 and Figure 1.

In the segments subjected to bleaching, the $\mathrm{Ca} / \mathrm{P}$ ratio decreased to $1.47,1.42$, and 1.70 for all three antioxidant treated groups as represented in Table 1 and Figure 1

After application of antioxidants, there seems to be reversal of mineral loss with increasing values similar to the baselines values represented as 2.14, 2.18, and 2.15 for three groups as represented in Table 1 and Figure 1.
Table 2: Tukey "honest significant difference" post hoc test showing statistical differences among sound, bleached, and antioxidant treated bleached enamel surfaces of all the three groups

\begin{tabular}{llll}
\hline S.no. & Enamel type & Variables & Significance \\
\hline \multirow{4}{*}{ Baseline } & Between groups & 0.000 \\
& Within groups & \\
& Total & \\
& Between groups & 0.000 \\
& Within groups & \\
& Total & \\
After antioxidant & Between groups 0.000 \\
& Within groups & \\
& Total \\
\hline
\end{tabular}

Table 3: Tukey "honest significant difference" post hoc test showing no statistical differences among all the three antioxidant groups for sound, bleached, and antioxidant treated bleached enamel surfaces

\begin{tabular}{llll}
\hline S.no. & Enamel type & Variables & Significance \\
\hline \multirow{4}{*}{ Baseline } & Between groups & 0.540 \\
& Within groups & \\
& Total & \\
& Between groups & 0.675 \\
& Within groups & \\
& Total & \\
After antioxidant & Between groups 0.243 \\
& Within groups & \\
& Total \\
\hline
\end{tabular}

The Tukey HSD post hoc test represented in Table 2 shows statistical differences among sound, bleached, and antioxidant treated bleached (AOTB) enamel surfaces of all the three groups.

The Tukey HSD post hoc test represented in Table 3 shows no statistical differences among all the three antioxidant groups for sound, bleached, and AOTB enamel surfaces.

There was a statistically significant decrease in the calcium/ phosphorus ratio for the bleached samples in comparison with the sound enamel. There was a comparable and significant increase in the calcium/phosphorus ratio values after application of the antioxidant with insignificant difference among the antioxidants evaluated in the study.

\section{Discussion}

The science of bleaching is developing rapidly and inseparable among the restorative fraternity. Various types of bleaching options are available among from vital and nonvital bleaching. In office, vital bleaching was found to have high clinical efficacy. The advantages and disadvantages of materials used for the bleaching procedures have to be analyzed thoroughly before their clinical usage. 
In the course of bleaching process, there is a considerable loss of minerals leading to changes in mechanical, physical, and chemical properties and in most of the clinical scenario requires the additional composites usage for the correction esthetic procedures. $^{24}$

The employment of composites immediately after a bleaching process is essential for the reversal of the above process. There is need to establish a balance between the minerals lost during the bleaching procedures with the antioxidants.

Employment of conventional antioxidant materials for the above purpose acted as a double-edged sword and had detrimental effects on the properties of the dental hard tissues.

The efficacy of antioxidant potential is multifactorial and can be categorized as: (a) substrate-related factors, (b) clinical techniquerelated factors, and (c) material-related factors. ${ }^{25-27}$

The substrate-related factors depend on the composition, compatibility, and resistance exhibited by the substrate toward capacity to replenishment of minerals lost during the bleaching process.

The interaction that occurs between the bleaching agents and substrate is related to fluid dynamics, viscosity of the bleaching agents, and the volume of the organic portion of the dental hard tissue. The organic content of enamel is very low in the range of $2 \%$ and is comparatively more in the range of $38 \%$ for dentin and exhibits difference in its behavior.

Bleaching agents have maximum interaction and attachment to dentin tissue molecules, reacting with the organic components. Additionally, substrate thickness and understanding the antioxidant dynamics play a vital role for the successful application of antioxidants.

According to Borges et al., maximum concentration of bleaching agents is concentrated in the outer dentin, which plays a key role in exhibiting the action and chance of adverse effect is directly proportional to it.

The clinical technique includes the measures and protocols taken to maximize the efficacy of antioxidants usage ranging from the duration of antioxidant, activation mechanism, and maintenance of aseptic field free from salivation.

The selection of antioxidants should be based on assessment of chemical nature, $\mathrm{PH}$, concentration, mechanism of action, indications, contraindications, and advantages and disadvantages related to the antioxidants.

According to Rashmi et al., ideal molecular weight of the antioxidant should be less than $500 \mathrm{~g} / \mathrm{mol}$ to remove the residual free radicals effectively.

The presence of hydroxylated structure aids in formation of complexes with less solubility on interactions with the proteins and carbohydrates and reduces the action on the nascent oxygen available after bleaching.

Contact time plays a significant role in efficacy of antioxidants reviewed. The ideal contact time depends on various material factors such as chemical nature and molecular size of antioxidants. The latency period following the bleaching and prior to the composite restoration was found to have no clinical significance implying the significance of antioxidants materials employed.

Most commonly used materials for the bleaching procedure include hydrogen peroxide, sodium perborate, and carbamide peroxide.

Bleaching agents release free radicals as nascent oxygen and hydroxyl ions when applied to dental structures. The oxidation process disintegrates the organic and inorganic elements and reduces the mechanical properties of dental hard tissues and morphological alterations in the inorganic and organic components. Electron-dense zones present in the dental stains act as the interceptive point for the lyses of large heavily stained particles to smaller less-stained particles. Additionally, it opens up the more pigmented carbon ring and converts them into carbon chains. When the carbon double-bond chains are converted to hydroxyl groups, the amount of light absorbed is reduced. Hence, the tooth appears lighter in color. Rotstein et al. indicated that bleaching agents cause changes in the levels of calcium and phosphorus among the hydroxyapatite crystals and increase the solubility leading to compromised mechanical strength properties. ${ }^{27}$

This leads to mobilization of calcium and phosphorus ions in the enamel surface leading to dentin exposure at cervical third of the tooth, resulting in hypersensitivity. Further leakage into the periodontal tissues leads to inflammatory resorption. ${ }^{28}$

Impact on the composite restoration occurs due to the residual oxygen interfering with the resin bonding; additionally, it inhibits polymerization that may cause bubble formation that further lowers the adaptation and penetration of enamel tags. ${ }^{29}$

The recommended latency period postbleaching ranges from 1 day to 6 weeks prior to the usage of composite restoration. To overcome this, various antioxidants ranging from conventional to natural antioxidants have been employed. ${ }^{30}$

The factors related to the antioxidant efficacy are related to the neutralization potential of free radicals generated during the bleaching along with the reestablishment of altered redox potential. Additionally the presence of singlet oxygen improves the antioxidant potential by providing the reducing and hydrogen ions.

The effects of various natural antioxidants such as guava seed extract, green tea extract, and pomegranate peel extract have been reported. ${ }^{31}$

Several studies concluded that the application of antioxidants after bleaching facilitates immediate bonding and restores mechanical properties to the enamel. ${ }^{24}$

In a previous study by Poorni et al., $10 \%$ sodium ascorbate solution showed a good reversal of the $\mathrm{Ca}-\mathrm{P}$ ratio in previous studies. Hence, the same concentration of sodium ascorbate was retained for this study.

To date, the effects of oregano as an antioxidant has not been evaluated in the literature that made us to conduct this study to minimize the adverse effect related to bleaching.

The mean calcium and phosphorus ratio of the enamel surface was detected using the EDAX. The principle employed in EDAX was collision of enamel specimens with electron beam, which leads to displacement of valence electron from the inner shell.

This vacancy was replaced by the transfer of electrons from the outer shell causing the generation of X-ray. The principle of EDAX analysis includes measurement of output of above process as graphic plot with peaks representing the energy values unique for an element and found to be helpful in identification of elements subjected for analysis.

The results of our study revealed the following values of mean calcium and phosphorus ratio of baseline enamel in group I, group II, and group III: 2.11, 2.20, and 2.09, respectively, as represented in Table 1 and Figure 1. Bleaching was done twice at an interval of 15 minutes.

After 24 hours, the samples were evaluated using EDAX and the mean calcium and phosphorus ratio was found to decrease from baseline values to $1.47,1.42$, and 1.70 , respectively, in all the three groups as represented in Table 1 and Figure 1. 
This might be attributed to the excessive dissolution of phosphorus ions which has weaker and covalent bonding to hydroxyapatite crystals than the calcium ions. The reason may be the weaker bonding of the phosphate moiety, which is covalently bonded to the hydroxyapatite crystals, than the calcium ions that are ionically bonded.

These results correlate with the previous study done by Santini et al. where bleaching leads to reduction of the phosphate ions which in turn resulted matrix degradation.

Immediate application of antioxidants after bleaching helps in maintenance of the mean calcium and phosphorus ratios which was found to be $2.14,2.18$, and 2.15 , respectively for all the three groups. This was comparable to that of baseline enamel values and was found to statistically insignificant. This result was probably due to oxygen cleansing activity of the antioxidants resulting in neutralization of free radicals.

Sodium ascorbate is a naturally occurring water-soluble antioxidant. It allows maintenance of the $\mathrm{Ca}$ and $\mathrm{P}$ ratio without dissolution of ions by restoring the altered redox potential of the substrate and thus reversing the reduced mineral ions. ${ }^{32}$

In a study conducted by Poorni et al., $10 \%$ sodium ascorbate was found to reestablish the baseline $\mathrm{Ca}-\mathrm{P}$ ratio of the bleached enamel.

Green tea is made from Camellia sinensis leaves. The main antioxidative ingredients present in green tea were catechins (GTCs) EC-6.4\%, Epigallo catechins (EGC-19\%), EC gallate (ECG-13.6\%), and EGC gallate (EGCG-59\%). EGCG is the most active and abundant catechin present in the green tea, which was found to have high water solubility and has the ability to destroy free radicals.

The antioxidant activity for green tea was related to the ability to neutralize the excess oxygen that exists after the bleaching, which reduces the dissolution of mineral ions and it restores the Ca-P wt\%. According to Ozelin et al., 10\% green tea extract had a better efficacy to completely neutralize the adverse effects. ${ }^{33}$

Oregano is a natural antioxidant herb native of dry rocks with a chemical structure of hydrocarbons and phenolic compounds. The antibacterial action is related to the presence of carvacrol and thymol. ${ }^{32}$

The proposed antioxidant mechanism of thymol is shown by the concentration-dependent decrease in cell number, degradation of cell morphology, and a decrease in the total protein content in an in vitro study. Thymol may impel cell death in human by apoptosis via mitochondrial pathways. ${ }^{34}$

The study was the first kind of study to assess the effect of oregano as a potent antioxidant in prevention of the alterations in mineral ions from the hydroxyapatite crystal.

\section{Limitations of the Present Study}

The sample size and results variation in the in vitro conditions require lots of clinical trials in human. Furthermore, it's an indirect way of evaluation of antioxidant efficacy. There was no valuable criterion to evaluate the mechanism of action of antioxidants employed. Future studies can compare and evaluate the efficacy of antioxidants against the remineralizing agents without causing any untoward effect on the substrate.

Future studies can be attempted to assess the efficacy of incorporation of antioxidants as inherent constituent of bleaching agents without compromising the efficacy. It has been claimed that the concentration, form, and mode of application play a pivotal role in the selection of materials.
Among the antioxidants evaluated in the study, it can be recommended to employ oregano as the material of choice due to its high efficacy with less adverse effect on the tooth substrate.

\section{Conclusion}

Within the limitations of the study, it can be concluded that the dissolution of $\mathrm{Ca}$ and $\mathrm{P}$ ions is due to the persistent peroxide effect after bleaching while bleaching with $35 \%$ hydrogen peroxide increases the dissolution of phosphorus ions and leads to the decrease the $\mathrm{Ca}-\mathrm{P}$ ratio with immediate application of antioxidants after bleaching effectively helps to maintain the $\mathrm{Ca}-\mathrm{P}$ ratio. The efficacies of all the three antioxidants such as $10 \%$ sodium ascorbate solution, green tea, and $5 \%$ oregano were found to be similar.

\section{References}

1. Viscio D, Gaffar A, Fakhry-SmithS, etal. Present and future technologies of tooth whitening. Compendium of continuing education in dentistry. Jamesburg, NJ 1995. Supplement. 2000(28):S36.

2. Prathap $\mathrm{S}$, Rajesh $\mathrm{H}$, Boloor VA, et al. Extrinsic stains and management: a new insight. J Acad Indus Res 2013;1(8):435-442.

3. Caneppele TM, Torres CR, Bresciani E. Analysis of the color and fluorescence alterations of enamel and dentin treated with hydrogen peroxide. Braz Dent J 2015;26(5):514-518. DOI: 10.1590/01036440201300249.

4. Leonard Jr RH, Bentley C, Eagle JC, et al. Nightguard vital bleaching: a long-term study on efficacy, shade retention, side effects, and patients' perceptions. J Esthet Restor Dent 2001;13(6):357. DOI: 10.1111/j.1708-8240.2001.tb01021.x

5. Abouassi T, Wolkewitz M, Hahn P. Effect of carbamide peroxide and hydrogen peroxide on enamel surface: an in vitro study. Clin Oral Investig 2011;15(5):673-680. DOI: 10.1007/s00784-010-0439-1.

6. Azrak B, Callaway A, Kurth P, et al. Influence of bleaching agents on surface roughness of sound or eroded dental enamel specimens. J Esthet Restor Dent 2010;22(6):391-399. DOI: 10.1111/j.17088240.2010.00372.x.

7. Ben-Amar A, Liberman R, Gorfil C, et al. Effect of mouthguard bleaching on enamel surface. Am J Dent 1995;8(1):29-32.

8. Bitter NC. A scanning electron microscopy study of the effect of bleaching agents on enamel: a preliminary report. Journal of Prosthetic Dentistry 1992;67(6):852-855. DOI: 10.1016/00223913(92)90600-f.

9. Bitter NC. A scanning electron microscope study of the long-term effect of bleaching agents on the enamel surface in vivo. Gen Dent 1998;46(1):84-88.

10. Cadenaro M, Navarra CO, Mazzoni A, et al. An in vivo study of the effect of a 38 percent hydrogen peroxide in-office whitening agent on enamel. J Am Dent Assoc 2010;141(4):449-454. DOI: 10.14219/jada. archive.2010.0198.

11. Ernst CP, Marroquin BB, Willershausen-Zönnchen B. Effects of hydrogen peroxide-containing bleaching agents on the morphology of human enamel. Quintessence Int 1996;27(1):53-56.

12. Güorgan S, Bolay S, Alacam R. In vitro adherence of bacteria to bleached or unbleached enamel surfaces. J Oral Rehabil 1997;24(8):624-627. DOI: 10.1046/j.1365-2842.1997.00534.x.

13. Haywood VB, Leech T, Heymann HO, et al. Nightguard vital bleaching: effects on enamel surface texture and diffusion. Quintessence Int 1990;21(10):801-804.

14. Hegedüs C, Bistey T, Flora-Nagy E, et al. An atomic force microscopy study on the effect of bleaching agents on enamel surface. J Dent 1999;27(7):509-515. DOI: 10.1016/s0300-5712(99)00006-8.

15. Hunsaker KJ, Christensen GJ, Christensen RP. Tooth bleaching chemicals-influence on teeth and restorations. In Journal of Dental Research 1990;69:303-303. 1619 Duke St, Alexandria, VA 22314: Amer Assoc Dental Research. 
16. Sa $Y$, Sun $L$, Wang $Z$, et al. Effects of two in-office bleaching agents with different $\mathrm{pH}$ on the structure of human enamel: an in situ and in vitro study. Oper Dent 2013;38(1):100-110. DOI: 10.2341/11-173-L.

17. Smidt A, Feuerstein O, Topel M. Mechanical, morphologic, and chemical effects of carbamide peroxide bleaching agents on human enamel in situ. Quintessence Int 2011;42(5):407-412.

18. Sun L, Liang S, Sa Y, et al. Surface alteration of human tooth enamel subjected to acidic and neutral $30 \%$ hydrogen peroxide. J Dent 2011;39(10):686-692. DOI: 10.1016/j.jdent.2011.07.011.

19. Titley KC, Torneck CD, Ruse ND. The effect of carbamide-peroxide gel on the shear bond strength of a microfil resin to bovine enamel. J Dent Res 1992;71(1):20-24. DOI: 10.1177/00220345920710010301.

20. Xu B, Li Q, Wang Y. Effects of $\mathrm{pH}$ values of hydrogen peroxide bleaching agents on enamel surface properties. Oper Dent 2011;36(5):554-562. DOI: 10.2341/11-045-1.

21. Josey AL, Meyers IA, Romaniuk K, et al. The effect of a vital bleaching technique on enamel surface morphology and the bonding of composite resin to enamel. J Oral Rehabil 1996;23(4):244-250. DOI: 10.1111/j.1365-2842.1996.tb00848.x.

22. Lai SC, Mak YF, Cheung GS, et al. Reversal of compromised bonding to oxidized etched dentin. J Dent Res 2001;80(10):1919-1924. DOI: $10.1177 / 00220345010800101101$

23. Vidhya S, Srinivasulu S, Sujatha M, et al. Effect of grape seed extract on the bond strength of bleached enamel. Oper Dent 2011;36(4): 433-438. DOI: 10.2341/10-228-L.

24. Coccimiglio J, Alipour M, Jiang ZH, et al. Antioxidant, antibacterial, and cytotoxic activities of the ethanolic Origanum vulgare extract and its major constituents. Oxid Med Cell Longev 2016;2016:1404505. DOI: 10.1155/2016/1404505.

25. Poorni S, Kumar RA, Shankar P, et al. Effect of $10 \%$ sodium ascorbate on the calcium: phosphorus ratio of enamel bleached with $35 \%$ hydrogen peroxide: an in vitro quantitative energy-dispersive X-ray analysis. Contemp Clin Dent 2010;1(4):223. DOI: 10.4103/0976$237 X .76388$.

26. Fearon J. Tooth whitening: concepts and controversies. J Ir Dent Assoc 2007;53(3):132-140.

27. Rotstein I, Lehr Z, Gedalia I. Effect of bleaching agents on inorganic components of human dentin and cementum. J Endod 1992;18(6):290-293. DOI: 10.1016/s0099-2399(06)80956-8.

28. Madison S, Walton R. Cervical root resorption following bleaching of endodontically treated teeth. J Endod 1990;16(12):570-574. DOI: 10.1016/S0099-2399(07)80199-3.

29. Kaya AD, Türkün $M$, Arici ME. Reversal of compromised bonding in bleached enamel using antioxidant gel. Oper Dent 2008;33(4): 441-447. DOI: 10.2341/07-115.

30. Gogia H, Taneja S, Kumar M, et al. Effect of different antioxidants on reversing compromised resin bond strength after enamel bleaching: an in vitro study. Journal of Conservative Dentistry: JCD 2018;21(1):100. DOI: 10.4103/JCD.JCD_325_16.

31. Sharafeddin F, Motamedi M, Modiri S. Effect of immediate application of pomegranate peel, grape seed and green tea extracts on composite shear bond strength of in-office bleached enamel. Res Biol Sci 2013;8:83-87.

32. Koparal AT, Zeytinoglu M. Effects of carvacrol on a human nonsmall cell lung cancer (NSCLC) cell line, A549. Cytotechnol 2003; 43(1-3):149-154. DOI: 10.1023/b:cyto.0000039917.60348.45.

33. Ozelin AA, Guiraldo RD, Carvalho RV, et al. Effects of green tea application time on bond strength after enamel bleaching. Braz Dent J 2014;25(5):399-403. DOI: 10.1590/0103-6440201300015.

34. Chang HT, Hsu SS, Chou CT, et al. Effect of thymol on $\mathrm{Ca}^{2+}$ homeostasis and viability in MG63 human osteosarcoma cells. Pharmacol 2011;88(3-4):201-212. DOI: 10.1159/000331864. 